\title{
L'ANDALOUSIE DU XIX SIÈCLE SOUS LES REGARDS DES VOYAGEURS
}

\section{Montserrat Serrano Mañes \\ Universidad de Granada}

\begin{abstract}
RÉSUMÉ: C'est au XIX siècle que l'Espagne s'impose aux voyageurs français comme lieu de destination à la mode : par l'influence des idées romantiques, mais aussi à cause des événements militaires du début du siècle. Quelques écrivains jouent un rôle d'éclaireurs : Laborde, Gautier, Dumas inscrivent littérairement une image de l'Espagne et de l'Andalousie que tous ceux qui les suivent cherchent à retrouver. Les regards des voyageurs témoignent du profond ancrage des topiques, et confirment la plupart du temps les récits de leurs devanciers. Leurs descriptions de l'Andalousie sont conditionnées par leurs lectures, et malgré les efforts de quelques-uns, ils la considèrent sous l'angle du dépaysement et du pittoresque transmis par leurs illustres prédécesseurs.
\end{abstract}

RESUMEN: En el siglo XIX, España se impone como destino de moda a los viajeros franceses, por influencia de las ideas románticas, y también a causa de los acontecimientos militares de principios de siglo. Algunos escritores -Laborde, Gautier, Dumas- introducirán en la literatura una imagen de España y de Andalucía que sus seguidores querrán reencontrar. Las miradas de esos viajeros son el testimonio de lo profundamente anclados que quedan los tópicos, y confirman en la mayoría de los casos los relatos de sus "maestros de viaje". Sus descripciones de Andalucía están condicionadas por sus lecturas, y pese a algunos esfuerzos aislados, todos la consideran bajo el mismo ángulo de extrañamiento y pintoresquismo transmitido por sus ilustres predecesores.

PALABRAS CLAVE: Siglo XIX, viajeros, tópicos, España, Andalucía.

MOTS CLÉS: le XIX siècle, voyageurs, topiques, Espagne, Andalousie. 
Si l'Espagne comme lieu de destination a été toujours privilégiée, c'est au XIX siècle que cette destination s'impose comme une mode aux voyageurs français. Sous l'influence des idées romantiques notamment, mais aussi à cause des événements militaires du début du siècle, l'Andalousie sera le but de toute une légion de voyageurs, guidés dans leur parcours par des écrivains qui ont joué le rôle d'éclaireurs : Laborde, Gautier, Dumas inscrivent littérairement une image de l'Espagne et de l'Andalousie que tous ceux qui les suivent cherchent à retrouver. Dans les textes de ces écrivains et « écrivants », les topiques foisonnent, les idées et les images de leurs illustres compatriotes sont reprises de manière insistante, et d'un récit à l'autre on voit se former une image de l'Andalousie aux couleurs changeantes, et pourtant immobile dans son essence scripturale ${ }^{1}$.

Si les années 1820-1850 sont celles où la fascination de la Péninsule est à son comble, de par l'influence indubitable des idées romantiques, l'intérêt de cette destination -celle de l'Espagne et surtout de l'Andalousie- se maintient au long du siècle, devenant presque un phénomène collectif ; l'affluence de touristes et de voyageurs se diversifie de plus en plus : les moyens de locomotion aussi, qui imposent une vitesse nouvelle au parcours, en même temps que l'exotisme tant recherché s'appauvrit.

Les regards portés au fil du siècle sur les villes visitées habituellement témoignent du profond ancrage des topiques, desquels on peut dégager une réalité partielle et partiale, et confirment la plupart du temps les récits de leurs mâ̂tres-en-voyage. L'entrée dans le pays de l'exotisme, effectuée par Despeñaperros, les conduit invariablement vers Cordoue, Grenade et Séville, le reste des villes étant considérées comme des destinations secondaires. Leurs descriptions ne varient guère d'un voyageur à l'autre, malgré les écarts temporels. Tout juste si l'on remarque parfois certaines nuances qui signalent des changements sociaux et économiques. Certes, ils ont des difficultés pour formuler d'une manière différente les paysages qu'ils sont venus retrouver et dont ils souhaitent vérifier la vérité. Leur perception du paysage, des villes, des hommes, est déjà formée et modelée, et ils sont presque toujours incapables d'échapper aux redites. Mais l'expérience du regard, forcément, s'inscrit dans les récits ${ }^{2}$. Le regard abolit les

1. D. Roche (2003:20) signale, à propos de l'utilisation des témoignages antérieurs par de nouveaux voyageurs, comment « Entre eux et leurs prédécesseurs se tisse un réseau, car ils se lisent, se copient, se corrigent, opposent leurs témoignages».

2. «S'il est une expérience à propos de laquelle la conscience du voyageur se trouve sollicitée, en particulier lorsqu'il a l'intention de faire de l'écriture une dimension supplémentaire de son périple, c'est évidemment celle du regard ». (Cogez, $2004: 81)$. 
limites entre celui qui regarde et l'objet contemplé. C'est pourquoi, si la description successive des villes-phares autochtones laisse une impression de déjà lu, de répétition et de redite, la personnalité des narrateurs transperce malgré tout les mots, remplissant l'espace qui va de la plume aux yeux avec la sensation de vécu. Les détails du décor, l'ordonnancement des repères spatiaux, diffère forcément des uns aux autres. L'accumulation ou gradation d'artifices change, et alors on perçoit la différence de regard. Ou bien, elle abonde sur l'égalité des témoignages, et nous, lecteurs, voyons se lever sous nos yeux une image de la ville immuable et immobile, égale à elle-même et drapée dans le stéréotype et le cliché autant que dans la vérité subjective du regard étranger qui lui est porté.

Les premières approches de cette Andalousie seront, par la force des choses, faites par des militaires. Leurs descriptions seront presque toujours sommaires car ils décrivent leur passage et les mouvements des troupes. Il existe cependant quelques exceptions : c'est le cas de John Rocca, deuxième mari de Mme. de Staël, laquelle a sans doute participé à la rédaction de ses mémoires. En tant que hussard, il a participé à la campagne d'Espagne entre 1808 et 1810. Poussé par Mme de Staël, il publie ses Mémoires de Guerre en 1815, dont le succès fut très vif : il fut édité en 1814 en Grande Bretagne, et réédité en France encore en 1908. Sa description de l'Espagne, plus littéraire que guerrière, fut appréciée et critiquée : les Espagnols lui reprochant de trop vanter les gloires de l'armée française, et les Français d'apporter une vision trop positive de l'Espagne et des Espagnols. C'est placé au pied de Sierra Morena que Rocca offre au lecteur sa vision du sud de la Péninsule, et elle ne semble pas s'écarter des clichés connus :

L'Andalousie est, sans contredit, la contrée de l'Espagne la plus fertile et la plus opulente par la nature. Il y a un proverbe dans les Castilles et dans la Manche, qui dit que l'eau seule du Guadalquivir engraisse plus les chevaux, que l'orge des autres pays. Le pain de l'Andalousie passe pour être le plus blanc et le plus exquis du monde entier, et les olives sont d'une grosseur surnaturelle. Le ciel de l'Andalousie est si serein et si pur, que 1'on peut y dormir presque toute l'année en plein air; on voit pendant l'été, et quelquefois même pendant l'hiver, des hommes couchés sous des portiques. Il y a une foule d'individus peu riches, qui voyagent sans s'inquiéter de chercher chaque soir des logements; ils portent leurs vivres avec eux, ou bien ils achètent des alimens (sic) que des femmes préparent pour les passans (sic), sur des réchauds, à l'entrée des grandes villes, ou sur les places publiques. Les pauvres ne se demandent pas, comme dans les pays du nord, s'ils ont une maison pour demeurer, mais, s'ils ont un bon manteau, qui puisse les protéger en été contre le contact immédiat des rayons du soleil, et les préserver des pluies pendant l'hiver.

On rencontre presqu'à chaque pas, en Andalousie, plus encore que dans les autres provinces de la péninsule, des traces ou des souvenirs des Arabes, et c'est le mélange 
singulier des coutumes et des usages de l'Orient avec les moeurs chrétiennes, qui distingue particulièrement les espagnols des autres peuples de l'Europe ${ }^{3}$.

Dans ces premières visions de l'Andalousie transmise par les Mémoires de militaires français de la même époque, la richesse des campagnes andalouses est une constante, ainsi que son rapprochement avec l'Afrique. Pour l'exemple, les Mémoires du général Cte. De Saint-Chamans, ancien aide de camp du maréchal Soult (1802-1832) : Mémoires typiquement militaires, peu descriptives, avec une vision générale de l'Espagne et du peuple espagnol très négative, où cependant transparaît l'émerveillement de l'auteur devant une campagne et un paysage surprenants à ses yeux. De son séjour à Séville, il gardera le souvenir d'une «province riche et civilisée », qui lui aura fait oublier pour un temps la guerre :

Je n'ai rien vu d'aussi beau dans aucun pays que cette campagne de l'Andalousie, qui offrait à la fois à nos yeux, étonnés de tant de richesses, les productions de l'Europe et de l'Afrique : ces bois d'orangers et de citronniers, ces buissons d'aloès et de lauriers-roses, ces forêts d'oliviers se mêlaient de la manière la plus pittoresque avec les arbres fruitiers, les vignes et les autres productions de notre pays. Cette population immense, qui ne nous paraissait avoir rien de l'air farouche des habitants des autres provinces espagnoles que nous avions parcourues, augmentait encore la riante perspective que nous avions devant nous, et, dans ces premiers moments, je crois que nous avions tous oublié les dangers et les horreurs de la guerre d'Espagne, et que peu d'entre nous auraient désiré être transportés en France ${ }^{4}$.

Le regard jeté par ces premiers visiteurs sur les villes andalouses diffère de celui que les écrivains romantiques vont mettre en place quelques années plus tard. La guerre aura favorisé un tourisme certes spécial, car forcé. Si pour Saint-Chamans Grenade, où il a séjourné pendant un temps chez l'habitant, ne mérite même pas une ligne, ce qui dit son manque d'intérêt pour le pays envahi et occupé, et Malaga le frappe par son climat - « Malaga est un des points les plus brûlants du climat de l'Espagne $»^{5}-$, pour le colonel Vigo Roussillon, qui commence sa campagne d'Espagne en 1810 -et dont le journal a été édité pour la première fois en 1981-, Cordoue, au milieu d'une campagne aussi riche et fertile que celle perçue par SaintChamans, a droit à un fragment de son journal de campagne pour donner une approche peu flatteuse de ses rues et de la mosquée-cathédrale, si célébrée par la suite :

3. J. Rocca, Mémoires sur la guerre des Français en Espagne, Paris : J. Gratiot, 1814, p. 174-176.

4. A. de Saint-Chamans, Mémoires du général Cte. De Saint-Chamans, ancien aide de camp du maréchal Soult (1802-1832), Paris, Plon, 1896, p. 167-168.

5. Ibid, p. 452. 


\section{L'ANDALOUSIE DU XIX SIÈCLE SOUS LES REGARDS DES VOYAGEURS}

Cette ville est bâtie dans une plaine magnifique et de la plus grande fertilité, au pied de la Sierra Morena, entre cette chaîne de montagnes et le Guadalquivir. Elle est, en général, mal construite quoique l'on y rencontre des palais et quelques belles maisons. Les rues sont étroites, tortueuses et mal pavées. On trouve dans tous les jardins et dans presque toutes les maisons, des fontaines distribuant les eaux que les Maures avaient été capter dans la montagne. Le monument le plus remarquable est la cathédrale. C'est une ancienne mosquée maure. Elle est couverte par une vaste terrasse que supportent trois cent cinquante colonnes de marbre ou de granit, mais elle manque d'élévation, ce qui nuit à la majesté de ce vaste édifice ${ }^{6}$.

Sa description est loin de celles des voyageurs qui suivront cette première vague de visiteurs forcés : Nul n'osera par la suite parler de ses rues en de tels termes -on préfèrera montrer une Cordoue silencieuse et presque morte, telle que Gautier $^{7}$ et Dumas ${ }^{8}$ l'ont décrite, sans reprendre la noirceur de leurs approches-, ni parler ainsi de sa mosquée.

Quelques années seulement plus tard, en 1823, Louis Viardot, écrivain de second ordre et doué d'un certain talent, fera son voyage en Espagne ; ses Souvenirs de chasse, publiés en 1849, sont déjà ceux d'un romantique, cherchant la couleur locale ${ }^{9}$; et elle coule à flots des fenêtres qui découvrent les patios de

6. Fr. Vigo Roussillon, Campagne 1793-1837 de François Vigo-Roussillon, grenadier de L'Empire, Paris : Éditions France-Empire, 1981, p. 298.

7. «Cordoue, autrefois le centre de la civilisation arabe, n'est plus aujourd'hui qu'un amas de petites maisons blanches par-dessus lesquelles jaillissent quelques figuiers d'Inde à la verdure métallique, quelque palmier épanoui comme un crabe de feuillage, et que divisent en îlots d'étroits corridors par où deux mulets auraient peine à passer de front. La vie semble s'être retirée de ce grand corps, animé jadis par l'active circulation du sang moresque ; il n'en reste plus maintenant que le squelette blanchi et calciné. » (Th.Gautier, Voyage en Espagne, Paris : Gallimard, 1981, p. 373-374).

8. Lequel donne une vision de la ville très négative, depuis son approche jusqu'à la description de ses rues. La description externe peint une ville banale : «...Cordoue, masse de maisons sans ombre, sans jardins, sans monuments autres que la cathédrale, Cordoue, malgré les trois ou quatre palmiers qui balancent au-dessus d'elle leurs gracieux éventails, Cordoue manque d'aspect ». (A.Dumas, Itinéraire de Paris à Cadix, Paris :Éds. François Bourin, 1989, p. 300). Dans la description interne transperce le mépris et le dégoût railleur : «Des rues étroites, sales, dans lesquelles il est défendu de jeter son eau, sans doute de peur que cette eau ne les lave quelque peu; des maisons basses et souvent d'un ton grisâtre, ce qui est si rare en Espagne, et grillées de haut en bas comme des prisons ; un seul monument dominant tout cela, la cathédrale : tel est le premier aspect de Cordoue. » (A.Dumas, Itinéraire de Paris à Cadix, Paris :Éds. François Bourin, 1989, p. 308).

9. En parlant des moines -l'aspect religieux étant l'un des thèmes qui reviennent dans les plumes de bon nombre de voyageurs-, il fait la remarque suivante : "Oh ! que vous me faites de la peine, m'écriai-je, en me montrant, à propos des moines, le revers de la médaille ! Ils sont si pittoresques, si couleur locale ! Voyez, quoi de plus beau, de plus original, que ce mélange bizarre d'hommes blancs, noirs, bruns, gris, bariolés, rasés ou barbus, tondus ou chevelus ? Qu'ils font bien dans les romans, les tableaux, les albums de touristes !» (L. Viardot, Souvenirs de chasse, Paris : Paulin et le Chevalier, 1848, p. 31). 
Séville, avec des images langoureuses de femmes échappées des contes des mille et une nuits :

Ces colonnes légères et gracieuses, ces lumières éclatantes, cette eau qui jaillit au milieu des fleurs et semble se mêler à la conversation par son murmure, ces femmes, légèrement vêtues, à demi couchées sous des orangers et des myrtes, tout cela forme un spectacle si nouveau, si magique, si digne des descriptions orientales de la princesse Scheherazade, qu'on peut se croire transporté, dans un songe des Mille et une Nuits, à la porte du paradis de Mahomet ${ }^{10}$.

Plus le siècle avance et plus les évocations des villes et paysages andalous se répondent en écho dans les récits des visiteurs. Les emprunts et les redites mettent en difficulté ces " écrivants », qui s'en tiennent aux témoignages littéraires laissés par leurs illustres devanciers tout en essayant de s'en éloigner ${ }^{11}$. La plupart ne pourront pas échapper à la médiocrité, l'essentiel étant déjà très connu des lecteurs et les connaissances et l'imagination manquant à bon nombre de ces voyageurs qui peu à peu se muent en touristes pressés. Les pôles référentiels resteront les mêmes pendant tout le siècle, ainsi que les clichés des empreintes arabes et de l'africanisation par exemple. Les apriori établis se maintiennent, les guides touristiques transmettent à côté des maîtres littéraires des itinéraires et des réflexions qui remplacent souvent l'observation directe : celle-ci n'est pas l'affaire du touriste distant et pressé. Pour l'exemple, Deux mois en yatch. Voyage aux côtes de l'Espagne, du Portugal et du Maroc ${ }^{12}$, du noble belge Camille de Renesse. Le récit, fruit d'après l'auteur des notes de voyage prises au jour le jour lors de son voyage de 1865 , raconte sous forme de lettres envoyées à une jeune fille son voyage au bord du steamer l'Intrépid, et ses visites à Cadix, Séville et leurs alentours. L'échappatoire choisie pour narrer consiste à s'épancher sur l'histoire des ces villes et à transmettre des opinions plus ou moins négatives aussi bien sur l'histoire de l'Espagne que sur les mœurs et le caractère du peuple espagnol, submergé dans l'ignorance et la superstition religieuse. Quand il s'agit de décrire, il s'en tient aux lieux communs. Ainsi, l'arabisation de Cadix accompagne la vision marine qui revient sous la plupart des plumes :

10. Viardot, L., Souvenirs de chasse, Paris : Paulin et le Chevalier, p. 18.

11. Comme le signale J.-R. Aymes (2003:266), « La grande affaire pour ceux qui viennent ensuite et qui, parce qu'ils suivent la mode et manquent d'imagination, vont se contenter d'emboîter le pas à ceux qui les ont précédés va être de se démarquer de ceux-ci, un tant soit peu ».

12. Publié en 1898, ce récit d'un dilettante connut un certain succès malgré sa médiocrité. Parti d'Ostende sur le yatch anglais de son cousin, le comte Monceau, le comte Camille Maximilien Frédéric de Renesse-Breidbach raconte sa traversée vers le Portugal et l'Espagne, et agrémente son bref séjour en Espagne de tous les topiques transmis par la littérature. 
Cadix cette presqu'île de pierre qui s'avance vers l'océan et que l'océan entoure de tous côtés, n'est reliée à la côte que par une langue de terre longue et étroite. Vue de la mer, elle a, sous le brillant soleil de l'Andalousie, l'aspect d'une ville orientale, avec ses murailles et ses maisons blanches à terrasses, que surmontent des clochers, des vigies, des phares, et d'innombrables belvédères qui ont l'air d'autant de minarets. Cadix a un climat merveilleux, le plus doux peut-être de toute l'Espagne, les ardeurs du soleil y étant presque toujours tempérées par la brise de la mer ${ }^{13}$.

Les regards portés par les peintres ne seront guère différents : l'Andalousie mauresque, au souvenir magnifié et faussé, s'oppose à celle d'une Espagne décadente. Salles Wagner, peintre de genre à succès, écrit à 81 ans ses souvenirs du voyage réalisé vers 1866, offrant dans Mémoires d'un vieil artiste (1896) des descriptions architecturales des villes andalouses. La description de Cordoue et de sa mosquée est agrémentée d'un aperçu historique qui choque avec l'état présent d'une ville délabrée, abandonnée et pauvre, transmise d'ailleurs par la plupart des visiteurs:

En parcourant cette ville déserte, vous rencontrez des façades sans édifices, où croissent la mousse et la mauve; des fenêtres sans vitres ni boiseries, où passent librement les oiseaux amis des grandes ruines ; des monastères inhabités, des temples déserts, des places où l'herbe croît, des rues silencieuses à toute heure, des marchés où l'on ne vend pas, des ateliers où l'on ne travaille pas, une population inactive, endormie, qui ne jouit plus des bienfaits de la civilisation de l'Islam, sans participer encore aux douceurs du progrès de notre époque ${ }^{14}$.

Joseph-Charles Vendryes, qui utilise aussi le pseudonyme éclairant de J.-Ch. Viator, écrit comme Salles Wagner très âgé ses souvenirs de voyages de jeunesse. En vrai voyageur, et non en touriste, il décrit vite et bien. Son voyage en Espagne, qu'il réalise en 1863, occupe une petite partie, mais savoureuse, de son livre Voyage en famille. Notes et souvenirs, publié quelques dix ans après. À côté des patios de Cordoue ${ }^{15}$, dont la description est devenue un lieu commun, des monuments et quartiers populaires de Séville, ou du regard désenchanté porté sur Grenade -« La

13. C. de Renesse-Breidbach, Deux mois en yatch. Voyage aux côtes de l'Espagne, du Portugal et du Maroc, Nice : Impr. des Alpes Maritimes, 1898, p. 94.

14. J. Salles Wagner, Mémoires d'un vieil artiste, Nîmes : Lavagne-Peyrot éditeur, 1896, p. 132.

15. « Nous parcourons la ville pour aller à la promenade de l'Alameda. Partout des balcons en fer forgé ; au rez-de-chaussée le patio, cour carrée semblable à l'atrium des maisons romaines ; autour règne une galerie couverte soutenue par des colonnes, un jet d'eau au centre, des orangers, des fleurs en profusion tapissent les murs, des meubles en cuir de Cordoue, des vases, des bibliothèques témoignent que c'est là que se passe la vie de l'Espagne : au-dessus une terrasse circulaire sur laquelle s'ouvrent les chambres à coucher. Rien n'est plus coquet, plus frais, plus ravissant que ces patios que nous devons retrouver partout en Andalousie » ( J.-Ch. Vendryes, Voyage en famille. Notes et souvenirs, Paris : Michel Lévy frères, 1874, p. 327). 
cathédrale de Grenade, quoiqu'on ait mis cent soixante ans à la bâtir, n'a rien de bien remarquable ; nous ne faisons qu'y entrer un moment en allant au MonteSagrado visiter les demeures des gitanos $\gg^{16}$-, nous trouvons l'approche de villes que d'autres voyageurs laissent à l'écart, et qui généralement ne sont pas visitées. Ainsi de Jaén, qui attrape l'attention du voyageur avec ses rues et ses maisons, et lui font évoquer une réalité non pas arabe -cliché banal du genre- mais moyenâgeuse :

Cette ville nous parut être un curieux type d'une cité du moyen âge, aux rues tranquilles et désertes ; nous en parcourons quelques-unes, étroites et tortueuses, aux maisons peintes en blanc, percées de rares ouvertures d'où retombaient en grappes épaisses, des plantes grasses à fleurs rouges conservées dans d'élégants vases d'Andujar. ${ }^{17}$

Certains se montrent donc plus originaux. Juliette de Robersart s'éloigne de l'image du touriste insouciant pour, depuis son séjour prolongé à Séville, donner des panoramas précis de l'Espagne et de la société andalouse qui reflètent sa connaissance et des villes et des gens. Malgré la persistance de quelques lieux communs avec lesquels, semble-t-il, tout voyageur se doit d'être d'accord, la sincérité de ses évocations renforce les qualités littéraires de son texte :

Le défilé de Despeña perros (sic) est ce que j'ai vu de plus remarquable jusqu'ici. Les montagnes de rochers s'entassent l'une sur l'autre, et forment une architecture gigantesque. La pierre est d'un rose baigné par soleil, qui m'a rappelé Saint-Jean de Latran ; un torrent mêle sa note grave à ce grave tableau. Les bandits, jadis, avaient choisi cet endroit pour repaire. On dit qu'il n'y en a plus ; j'aime à ne pas le croire. D'ailleurs, les gendarmes garnissaient la route de distance en distance ; ce n'était pas pour nous arrêter, à coup sûr.

(...)

La végétation change ; voici le sud, presque l'Afrique. Les cactus et les aloès bordent la route ; j'aime mieux l'aloès sous notre ciel gris, et triste prisonnier dans sa caisse ; il est dépaysé dans son pays. Cette plante dégingandée et terne, compose une assez bonne harmonie avec l'olivier. L'olivier, gloire injuste et mal acquise, n'est qu'un saule poudreux dont toutes les feuilles semblent tachées d'huile. ${ }^{18}$

Le récit de son premier voyage, réalisé en 1863, est le résultat de la compilation de sa correspondance avec plusieurs destinataires, surtout avec son amie

16. Id., p. 318.

17. Id., p. 316.

18. J. de Robersart, Lettres d'Espagne. Paris : Victor Palmé, 1866, p. 21. 
Charlotte de Grammont. La vérité de ses observations, la vivacité de sa plume, les récits de ses aventures sur le sol espagnol accordent à ses Lettres d'Espagne, parues en $1866^{19}$, des couleurs d'originalité. La peinture de Séville, où elle habite chez des parents, s'enrichit de visites non seulement culturelles -couvents, églises, monuments...- mais aussi sociales : ses promenades, ses relations. Les touches descriptives sont volontairement légères, dans sa volonté affichée d'épargner à ses destinataires les lieux communs trop connus. C'est ainsi qu'elle escamote de possibles longues dissertations sur la ville :

Les rues sont blanches, étroites, enlacées, mauresques, peintes à la chaux ; elles me rappellent le grand béguinage de Gand. Mais je m'arrête court comme une poupée à ressort. Les descriptions, ça ennuie tout le monde. Causons : Point d'insectes ; grande, grande propreté partout. La hoye, toujours pour premier plat ; on l'appelle ici el cocido, ou puchero, ou olla. ${ }^{20}$

Sachant très bien quelles sont les peintures à éviter et où réside la nouveauté, elle se permet de temps en temps des descriptions très personnelles des villes les moins connues. Ronda occupera un lieu de choix, son paysage étant fait pour frapper les esprits épris de magnificence naturelle :

Après huit heures d'une marche ininterrompue, faite plutôt pour les chamois et les daims que pour les hommes, j'ai aperçu Ronda à l'horizon. J'étais ivre de fatigue, mais quel but digne de la route, quel nid d'aigle fièrement campé sur les deux rocs, bastions gigantesques de cette forteresse, et séparés l'un de l'autre par une déchirure de plusieurs centaines de pieds ! Au fond coule un torrent ; il rugit, il sort de ses ténèbres furieux, se roule de roc en roc, d'étage en étage, dans un dernier précipice, fumant et bondissant. Cela s'appelle el Taxo. Un pont, bâti à cet endroit, réunit la ville depuis une centaine d'années. Il a deux arches placées l'une au dessus de l'autre. Je suis descendue au bas ce matin. La vue des précipices attire et repousse, donne le vertige et l'ivresse. Celui-ci est tapissé de lierres, de fleurs mouvantes, et ses parois sont d'une brèche rose veinée. ${ }^{21}$

Dans les décennies suivantes, les voyageurs s'adapteront aux nouveaux moyens de voyage, tout en conservant et itinéraires et idées reçues. Séville et sa rue des Sierpes, Grenade avec l'Alhambra et le Sacromonte, Cordoue avec sa

19. Elle revient en Espagne en 1876, et maintient toujours une correspondance avec Charlotte de Grammont. Les deux voyages paraîtront dans l'édition de 1879 : Lettres d'Espagne, par la comtesse Juliette de Robersart, Nouvelle édition, considérablement augmentée, Paris, Watelier libraire-éditeur ; Lille, imprimerie Saint Augustin ; Bruges: Desclée de Brouwer et Cie., 1879.

20. J. de Robersart, Lettres d'Espagne. Paris : Victor Palmé, 1866, p. 27.

21. Id., p. 146. 
mosquée et son silence insistant, reviendront sous les écritures de ces visiteurs. La plume d'un Robida ${ }^{22}$, alerte et pleine de fraîcheur, peindra de son trait ferme et léger les rues de Séville avec de nouvelles couleurs ${ }^{23}$, et celles de Grenade parâ̂tront aux yeux des lecteurs sillonnées par un passant attentif :

En sortant du bois à la porte des grenades, on descend en ville par la côte de Gomelès, une très jolie rue aux grandes maisons riantes, habitée jadis par la tribu des Gomelès, ennemie avec les Zegries du parti des Abencérages. Au bas de la cuesta de los Gomelès, on se trouve au plein coeur de la ville. Sur la gauche commence le Zacatin, la vieille rue marchande, en face est la Plaza Nueva et sur la droite serpente le Darro profondément encaissé, bordé de maisons à pic. ${ }^{24}$

Les voyageurs, qui ne fréquentent presque plus que les chemins de fer, voient les paysages défiler sous leurs yeux, et les villes se dépouiller de leurs charmes anciens. Les cactus et les aloès sont toujours omniprésents, l'évocation africaine toujours de mise. Pour Henri de Rothschild, qui en 1889-1890 fait le voyage en Espagne commodément en famille, Séville est une ville presque orientale - « Rien de plus joli que ces rues de Séville, rien de plus original et de moins européen. Il est vrai que l'on est à plus de deux mille kilomètres de Paris ; mais on se croirait, ou à Alger, ou même à Jérusalem $»^{25}$, et malheureusement, les petites maisons de Grenade, malgré leur cachet hispano-arabe, « ont perdu presque entièrement le caractère andalou $»^{26}$. Le regard s'assombrit quand la réalité recherchée ne se montre plus à leurs yeux. D'autres touristes manifesteront, comme André Tandonnet, leur dégoût pour une modernisation qui nuit à la couleur locale et au pittoresque tant recherché, ou bien comme Théophile Roller reflèteront dans leurs écrits des villes dépourvues de grâce. Si Cordoue, dont les maisons sont « dignes extérieurement d'une obscure province », vaut encore quelque chose, c'est par ses

22. Albert Robida, dessinateur, aquarelliste, caricaturiste, journaliste et romancier, voyage en Espagne en 1879 et publie une année plus tard Les vieilles villes d'Espagne. Notes et souvenirs.

23. « La calle de las Sierpes est une rue très étroite qui va depuis la poste, derrière la place del Duque, jusqu'à la place de la Constitution où se trouve l'ayuntamiento. Au-dessus de ses maisons bariolées, peintes en jaune, en rose et même en bleu tendre, la rue est entièrement couverte d'un bout à l'autre par des toiles accrochées aux toits, aux carrefours les toiles s'entrecroisent de façon à ne laisser passer aucun rayon indiscret, et l'on n'y jouit que d'une petite chaleur d'étuvée très supportable au lieu de rôtir comme dans les rues dépourvues d'abris » (196).

24. A. Robida, Les vieilles villes d'Espagne. Notes et souvenirs, Paris : Maurice Dreyfous, 1880, p. 266.

25. H. de Rothschild, Souvenirs d'Espagne. Avril 1889-mars 1890, Mâcon : Imprimerie de Protat frères, p. 35.

26. Ibidem., p. 91-92. 
patios, qui gardent pour ce touriste mal informé leur charme mauresque. Mais Grenade a été trop vantée, et seule l'Alhambra mérite encore ses faveurs :

Malgré sa réputation et son nom poétique, Grenade est bien la plus malpropre et la plus impraticable -quand il pleut- de toutes les petites villes de province. Je ne sais ce que l'édilité y était du temps des Maures, mais il me paraît certain que, si le roi Boabdil avait dû s'enfuir à travers les cloaques de fanges qui encombrent la cité et ses faubourgs, il y serait resté englué. À part deux ou trois couvents à portiques majestueux, la cathédrale d'un classicisme prétentieux, les tombeaux de Ferdinand vainqueur des Maures, et d'Isabelle la Catholique, les ruelles de l'Albaycin et les grottes des Gitanos, il n'y a que l'Alhambra à visiter à Grenade. Mais c'est bien quelque chose, et ce monument seul vaudrait le voyage en Espagne. Il est encadré, d'ailleurs, dans un paysage plein de grandeur, entre la Vega ou plaine verdoyante et les cimes neigeuses de la Sierra Nevada. ${ }^{27}$

Rien de trop différent dans les derniers regards féminins du siècle. En 1880, Noémi Cadiot visite l'Andalousie et, cachée sous le pseudonyme de Claude Vignon, publie un peu plus tard Vingt jours en Espagne. C'est l'époque du règne d'Alphonse XII, et les éléments qu'elle recherche sont toujours les mêmes : visites artistiques et monumentales, mœurs étrangères. Son attention est attirée à Séville par le déjà lu de la calle Sierpes, mais aussi sur les habitudes des femmes sévillanes -«Quand il leur faut traverser une échappé de soleil, les femmes s'abritent de leur éventail et passent vite, comme des Parisiennes qui traversent le boulevard ${ }^{28}-$, à Grenade par le quartier gitan et l'Albaycin, où triomphent les tons sombres évoqués par les écrivains romantiques. Mais le charme ancien n'opère plus, en cette fin de siècle, et Grenade n'est plus que par l'Alhambra :

En dehors de l'Alhambra, Grenade n'offre plus grand intérêt. Séville est une capitale ; Grenade est une ville de province qui de plus, chaque jour, devient une ville morte.

Le Zacatin, jadis quartier commercial et animé, est morne et pauvre. Hormis des couvertures de laine brodée et des capes façonnées que les Espagnols ne portent plus et qui ne semblent plus avoir d'emploi pratique je ne vois pas ce qu'on pourrait acheter dans le Zacatin. Quant au bazar mauresque, s'il est entier et très intéressant par sa conservation, il est par son abandon et sa solitude, plus triste qu'un cimetière. $^{29}$

27. Roller, Th. (1887) Lettres d'Espagne, Alençon : Impr. Typographique F. Guy, p. 26.

28. Cl. Vignon, Vingt jours en Espagne, Paris : Calman-Lévy, 1881, p. 32.

29. Ibid., p. 54-55. 
À l'extrême pointe du siècle, en 1899, une autre femme, Maria Star, suit un parcours géographique semblable. Les contours des villes, cependant, s'estompent. L'évocation de Séville ou de Grenade et de leurs monuments est légère - « La ville de Grenade est très vaste. Nous la parcourons dans tous les sens ${ }^{30}-$, l'écrivain épargne des images de décadence ou des redites qui ne sont plus en cours, et si Cadix garde son charme, c'est par des éléments naturels qui évitent des arrêts descriptifs sur la ville :

\begin{abstract}
À peine arrivés, nous nous sommes promenés sur les bastions garnis d'échauguettes, dont les profils guerriers se découpaient en clair sur un ciel bleu sombre, éclaboussé d'étoiles. La transparence de ce ciel était inouïe malgré l'heure tardive, et la brise de mer était si douce que j'aurais voulu prolonger cette minute exquise et passer la nuit à la belle étoile ${ }^{31}$.
\end{abstract}

Mais un esprit différent habite ces deux femmes, qui pourraient à elles seules symboliser les deux visages des voyageurs d'outre-Pyrénées comme de tout voyageur : face au désir de retrouver les rivages connus, qui cache à peine le mépris de l'autre, de l'inconnu -c'est Vignon lançant, à la fin de son récit, son cri de soulagement : «Ma foi ! Oui, c'est un beau pays que la France !...même quand on revient de Suisse et d'Italie ; surtout quand on revient d'Espagne ! $\gg^{32}-$, la volonté de dépaysement comme seul moyen de comprendre l'autre exprimé par Maria Star : «Il me semble que la philosophie du voyage est de savoir s'adapter aux coutumes, aux mets et aux boissons du pays; vouloir les transporter ailleurs ou importer ses habitudes, est presque une erreur, presque une imprudence ${ }^{33}$.

On le voit, les bornes du voyage ne varient pas, malgré les progrès techniques que ces tout nouveaux touristes laissent entrevoir dans leurs itinéraires : des mulets et de la diligence au chemin de fer, les choses changent. Mais ce sont toujours les mêmes villes -Cordoue, Grenade, Séville, Cadix, Malaga-, qui passent sous leurs yeux. Les regards successifs portés sur l'Andalousie et sur ses villesphare offrent un kaléidoscope aux couleurs vives, aux formes changeantes et nonobstant attendues. Hélas, les habitants, les coutumes, les paysages ne changent guère, les topiques sont fortement enracinés dans les regards portés sur ce

30. E. de H. Stern, Impressions d'Espagne, Paris : Soc. d'Éditions littéraires et artistiques/P. Ollendorf, 1900, p. 138.

31. Ibid., p. 146.

32. Op. cit., p. 65.

33. Op. cit., p. 49. 
pays qui se doit d'être exotique et arriéré. Les coups d'œil sur ces lieux, sur leurs gens, ne font que confirmer l'idée que le voyageur ne peut jamais se contenter d'enregistrer la réalité, et que "Toute représentation écrite obéit en effet à des conventions scripturales $»^{34}$.

Ces voyageurs, « écrivants » plus qu'écrivains pour la plupart, jetant dans leurs récits destinés à leurs proches ou à un nombre assez restreint de lecteurs leurs souvenirs de voyage, cherchent les plaisirs du déjà su, ou du déjà lu : une Andalousie exotique, semi africaine et mauresque ${ }^{35}$, des parcours semés de dangers qui donnent tout juste des frissons de peur : des bandits et des brigands, des auberges mal entretenues, des moyens de transport incommodes, des désagréments qui confirmeront ce qu'ils ont lu et qu'ils pourront raconter comme des expériences propres $^{36}$. Peu d'entre eux essayeront de faire le partage entre les idées reçues et la réalité, car l'appétit d'exotisme s'unit à la force descriptive des tout premiers narrateurs pour imposer une vision des choses qu'ils veulent immuable. Leurs regards sont conditionnés par leurs lectures, et malgré les efforts de quelques-uns, ils considèrent l'Andalousie -ses villes, ses paysages, ses gens- sous l'angle du dépaysement et du pittoresque transmis par leurs illustres devanciers.

\section{Bibliographie}

ANTOINE, Ph. (1997) Les récits de voyage de Chateaubriand, Paris : Honoré Champion.

AYMES, J.-R. (1983) L'Espagne romantique (Témoignages de voyageurs français), Paris : Eds. A.M. Métailié.

AYMES, J.-R. (2003) Voir, comparer, comprendre, Paris : Presses Universitaires Sorbonne.

COGEZ, G. (2004) Les Écrivains voyageurs au XX siècle, Paris : Seuil.

DUMAS, A. (1848) Itinéraire de Paris à Cadix, Paris : Éds. François Bourin, 1989.

34. Ph. Antoine, Les récits de voyage de Chateaubriand, Paris : Honoré Champion, 1997, p. 10.

35. J.-R. Aymes (1983:16) signale comment « La véritable Espagne, selon le critère des voyageurs romantiques, est donc andalouse, c'est-à-dire semi-africaine ».

36. G. Cogez (2004:17) formule très bien cette difficulté en parlant de Gautier, modèle de la plupart des voyageurs du XIX siècle à côté de Mérimée, Laborde ou Dumas : «Difficulté liée d'abord au fait que l'on peut être confronté à des réalités très différentes de celles qu'on a l'habitude de nommer, et difficulté aussi à trouver des formulations neuves pour ne pas répéter ce qu'ont déjà dit sur le même sujet les précédents voyageurs ». 
GAUTIER, Th. (1843) Voyage en Espagne, Paris : Gallimard, 1981.

RENESSE-BREIDBACH, C. de (1898) Deux mois en yatch. Voyage aux côtes de l'Espagne, du Portugal et du Maroc, Nice : Impr. des Alpes Maritimes.

ROBERSART, J. de (1866) Lettres d'Espagne. Paris : Victor Palmé.

ROBIDA, A. (1880) Les vieilles villes d'Espagne. Notes et souvenirs, Paris : Maurice Dreyfous.

ROCHE, D. (2003) Humeurs vagabondes, Paris : Fayard.

ROLLER, Th. (1887) Lettres d'Espagne, Alençon : Impr. typographique F. Guy.

ROTHSCHILD, Henri de (1890) Souvenirs d'Espagne. Avril 1889-mars 1890, Mâcon : Imprimerie de Protat frères.

SALLES WAGNER, Jules (1896) Mémoires d'un vieil artiste, Nîmes : LavagnePeyrot éditeur.

STERN, E. de H. (1900) Impressions d'Espagne, Paris : Soc. d'Éditions littéraires et artistiques/P. Ollendorf).

VENDRYES, J.-Ch. (1874) Voyage en famille. Notes et souvenirs, Paris : Michel Lévy frères.

VIARDOT, L. (1848) Souvenirs de chasse, Paris : Paulin et le Chevalier.

VIGNON, Cl. (1881) Vingt jours en Espagne, Paris : Calman-Lévy.

VIGO ROUSSILLON, Fr. (1981) Campagne 1793-1837 de François Vigo-Roussillon, grenadier de L'Empire, Paris : Éditions France-Empire. 